The Astronomical Journal, 119:1111-1122, 2000 March

(C) 2000. The American Astronomical Society. All rights reserved. Printed in U.S.A.

\title{
THE SPECTRA AND ENERGIES OF CLASSICAL DOUBLE RADIO LOBES
}

\author{
Katherine M. Blundell and Steve Rawlings \\ Astrophysics, Nuclear and Astrophysics Laboratory, University of Oxford, Keble Road, Oxford, OX1 3RH, England, UK \\ Received 1999 August 16; accepted 1999 December 16
}

\begin{abstract}
We compare two temporal properties of classical double radio sources: (1) radiative lifetimes of synchrotron-emitting particles and (2) dynamical source ages. We discuss how these can be quite discrepant from one another, rendering use of the traditional spectral aging method inappropriate: we contend that spectral ages give meaningful estimates of dynamical ages only when these ages are $\ll 10^{7} \mathrm{yr}$. In juxtaposing the fleeting radiative lifetimes with source ages that are significantly longer, a refinement of the paradigm for radio source evolution is required. We move beyond the traditional bulk backflow picture and consider alternative means of the transport of high Lorentz factor $(\gamma)$ particles, which are particularly relevant within the lobes of low-luminosity classical double radio sources. The changing spectra along lobes are explained, not predominantly by synchrotron aging but by gentle gradients in a magnetic field frozen into a low- $\gamma$ matrix that illuminates an energy distribution of particles, $N(\gamma)$, controlled largely by classical synchrotron loss in the high magnetic field of the hot spot. A model of a magnetic field whose strength decreases with increasing distance from the hot spot and in so doing becomes increasingly different from the equipartition value in the head of the lobe is substantiated by constraints from different types of inverse Compton scattered X-rays. The energy in the particles is an order of magnitude higher than that inferred from the minimum energy estimate, implying that the jet power is of the same order as the accretion luminosity produced by the quasar central engine. This refined paradigm points to a resolution of the 1994 findings of Rudnick et al. and Katz-Stone \& Rudnick that both the Jaffe-Perola and Kardashev-Pacholczyk model spectra are invariably poor descriptions of the curved spectral shape of lobe emission and, indeed, that for Cygnus A all regions of the lobes are characterized by a "universal spectrum."
\end{abstract}

Key words: galaxies: active — galaxies: evolution — galaxies: jets — quasars: general — radio continuum

\section{INTRODUCTION: LOBE LOSS MECHANISMS}

That the radio radiation from classical double radio sources arises from synchrotron emission is beyond doubt: the spectra of many emitting regions of these sources are predominantly power-law and often characterized by a high fractional polarization. Synchrotron cooling is by no means the only significant loss mechanism to be manifested on the spectra of these sources, however. A recent study has demonstrated the importance of expansion losses for evolving radio sources, which we briefly review.

Blundell, Rawlings, \& Willott (1999, Fig. 8) found that the strongest correlation ${ }^{1}$ between any two properties of low-frequency-selected radio sources in complete samples is that sources with longer linear sizes $D$ have steeper spectra $\alpha$ when these are evaluated at rest-frame $151 \mathrm{MHz}$. This is known as the $D-\alpha$ correlation. This correlation is independent of any separate correlation between other source properties: the Spearman rank correlation coefficient at constant luminosity $P$ and constant redshift $z$ is $r_{D \alpha \mid P z}=$ 0.37 , with any dependence on the assumed cosmological model only arising in the significance of this effect (which varies between 5 and $6 \sigma$ for the models considered). Moreover, simulations of model sources discussed in that paper show that throughout the lifetime of an individual source, its low-frequency spectrum steepens. These simulations,

\footnotetext{
${ }^{1}$ The strongest intrinsic correlation, that is; a very strong artificial correlation is found between luminosity and redshift because of the Malmquist bias.
}

when combined with the sampling functions due to the survey flux limit and light-cone interception, regenerate the $D-\alpha$ correlation.

Blundell et al. (1999) attributed the steepening of lowfrequency spectra with age to adiabatic expansion losses. Adiabatic expansion losses occur when an emitting blob of plasma expands, with a consequent decrease in the magnetic field strength and in the energies of the particles themselves (Scheuer \& Williams 1968). When one considers a fixed observing frequency, a lower magnetic field means that higher Lorentz factor particles are required to radiate at the chosen frequency. The relationship between the Lorentz factor $\gamma$ of particles giving rise to emission at some specific frequency $v$ and the ambient magnetic field strength $B$ is given by

$$
\gamma=\left(\frac{m_{e}}{e B} 2 \pi v\right)^{1 / 2},
$$

where $e$ is the charge on an electron and $m_{e}$ is its rest mass.

Because it is essential to factor in not just synchrotron losses (and, at high redshift, inverse Compton losses off the cosmic microwave background), but also adiabatic expansion losses to analyses of the spectra of radio lobes, we consider in this paper how these arise and what changes must be made to existing views of radio source evolution. The outline of this paper is as follows: In $\S 2$ we describe briefly studies to date that have inferred spectral ages of classical double radio sources. In $\S 3$ we specify and quantify the radiative lifetimes of synchrotron particles in the lobes. In $\S 4$ we discuss what is meant by the lifetime of a radio 
source, and then in $\S 5$ we compare the dynamical ages of sources we observe with their spectral ages and with the relevant radiative lifetimes. In $\S 6$ we revisit the problem, first identified by Jenkins \& Scheuer (1976), that longer and less powerful radio sources invariably have lobe emission (even at $\mathrm{GHz}$ frequencies) that often persists back to the core, while shorter and more powerful sources do not have lobe emission extending back to the core. We discuss seven mechanisms in $\S 7$ by which the findings of the two previous sections might be reconciled. One of these mechanisms has been almost completely neglected in the past and is worthy of further serious consideration. We discuss possible information that might be gleaned from the rarity of "dead" radio galaxies, discussed in $\S 8$. In $\S 9$ we discuss a refined picture of radio source growth.

Our refined model enables us to identify a contributionmore important than just synchrotron cooling - to spectral steepening that is observed to increase along radio lobes with increasing distance from the hot spot; this we discuss in $\S 10$. In $\S 11$ we investigate independent support for the predictions of this model from detections of inverse Compton X-rays associated with radio sources and consider the implications for the overall energy budget.

We examine the results of Rudnick, Katz-Stone, \& Anderson (1994) and Katz-Stone \& Rudnick (1994) in $\S 12$ in the light of the model discussed in $\S 9$. We conclude in $\S 13$.

\section{SPECTRAL AGING}

If a magnetized blob of plasma, with an initial power-law spectrum $N(\gamma)$ to infinite energy, were subject only to synchrotron cooling via the uniform magnetic field strength $(B)$ in which it is deemed to be immersed, then a cutoff or "break" in that power-law spectrum would appear (Kardashev 1962; Pacholcyzk 1970; Jaffe \& Perola 1973). The frequency at which this break occurs $\left(v_{b}\right)$ would move to lower frequencies as $v_{b} \propto B^{-3} t^{-2}$, where $t$ is the time elapsed since the blob of plasma had its particles accelerated to their initial power-law energy distribution. Fits to the curved spectra observed in small transverse elements of radio lobes have led to changes in spectral curvature to be identified with these break frequencies. These estimates of the break frequencies, together with an estimate of the magnetic field strength, have led to estimates of the "spectral age" (or time elapsed since acceleration) being made across successive elements in the lobes of radio sources (e.g., Winter et al. 1980; Myers \& Spangler 1985; Alexander \& Leahy 1987; Liu, Pooley, \& Riley 1992). These workers have interpreted the spectral ages of the regions of lobe nearest the core as being closely related to the source age.

However, if other loss mechanisms besides synchrotron cooling, such as adiabatic expansion losses as discussed in $\S 1$, are also taking place then the observed break frequency in the spectrum will have been reached more rapidly than the above simple picture will allow, as long as the magnetic field strength has only tended to decrease with time. Unless the true value of the magnetic field is vastly less than that estimated (see discussion in $\S 7.1$ ), this renders simple spectral ages to be overestimates of the time elapsed since acceleration (see, e.g., Alexander 1987). It is likely that a given blob of plasma will have spent some time in a higher magnetic field when it was near the hot spot than subsequently, for example, and knowledge of the "history" of the magnetic field evolution is required to begin to reinterpret mea- sured spectral ages. A modification of the formula for break frequency when both synchrotron and adiabatic expansion losses take place may be found in Blundell \& Alexander (1994).

Many have aired concerns about the spectral aging method. Jones, Ryu, \& Engel (1999), for example, have pointed out that any mixing of particles within the lobes will contaminate estimates of spectral ages, while Tribble (1993) has demonstrated that subtleties in spectral evolution are more appropriately attributed to the magnetic field configuration than to differences in the evolution of the pitch-angle distribution, while others have discussed the difficulty of estimating the magnetic field strengths or identifying the magnetic field history for a given blob of plasma (e.g., Siah \& Wiita 1990; Wiita \& Gopal-Krishna 1990; Rudnick et al. 1994; Rudnick 2000), and we do not repeat them here. We remark, however, that unless the spectral ages to be measured are much shorter than the radiative lifetimes of the particles that constitute the evolving spectrum, spectral aging is a flawed method.

\section{RADIATIVE LIFETIMES}

We now consider for how long a synchrotron particle can contribute to emission observed from a radio lobe.

A very naive estimate of the length of time for which a synchrotron-emitting electron will radiate under the conditions thought to be found in a lobe of a classical double radio source may be obtained by dividing the total kinetic energy $E$ of one electron by its synchrotron power $P$, as follows:

$$
\frac{E}{P}=\frac{(\gamma-1) m_{e} c^{2}}{2 \sigma_{\mathrm{T}} c \gamma^{2}(v / c)^{2} U_{\operatorname{mag}} \sin ^{2} \theta} .
$$

Here $\gamma$ is the Lorentz factor of the electron, $c$ is the speed of light, $\sigma_{\mathrm{T}}$ is the Thomson scattering cross section for electrons, $v$ is the velocity of the electron, $U_{\text {mag }}$ is the magnetic energy density, and $\theta$ is the pitch angle of the electron's motion with respect to the magnetic field lines. We set $v / c$ and $\sin ^{2} \theta$ to 1 . Equation (1) tells us that to radiate at restframe $5 \mathrm{GHz}$ in a magnetic field strength of $1 \mathrm{nT}$ the required Lorentz factor of the electron is $\gamma \sim 10^{4}$.

The radiative lifetime of such an electron would be just under $10^{7}$ years according to equation (2) with $\gamma=10^{4}$. However, this naive sum overlooks two important factors: (1) Clearly a particle with $\gamma \sim 10^{4}$ cannot radiate for $10^{7} \mathrm{yr}$ at $5 \mathrm{GHz}$ in the assumed constant magnetic field. Rather, this duration of time indicates how long it would take a particle cooled by synchrotron losses to change from having $\gamma \sim 10^{4}$ to having $\gamma \sim 1$ if its cooling rate were maintained at the level expressed in equation (2). (2) The lobe of a radio source is an evolving entity, and the adiabatic expansion losses (especially the decreasing magnetic field) discussed earlier mean that particles with continuously higher $\gamma$ are required to produce the emission at the same chosen frequency as the source ages. For example, in the scenario above, a $\gamma \sim 10^{4}$ particle will emit most of its radiation at 5 $\mathrm{GHz}$ if the magnetic field strength is $1 \mathrm{nT}$. If the field lowered adiabatically to $0.1 \mathrm{nT}$, then particles whose Lorentz factors are $3 \times 10^{4}$ are required to produce the emission at the same "observing frequency" of $5 \mathrm{GHz}$. In a radio lobe, changes in the magnetic field go hand in hand with changes in the energies of the particles themselves since it is the expansion of the lobe that governs both. Thus the 


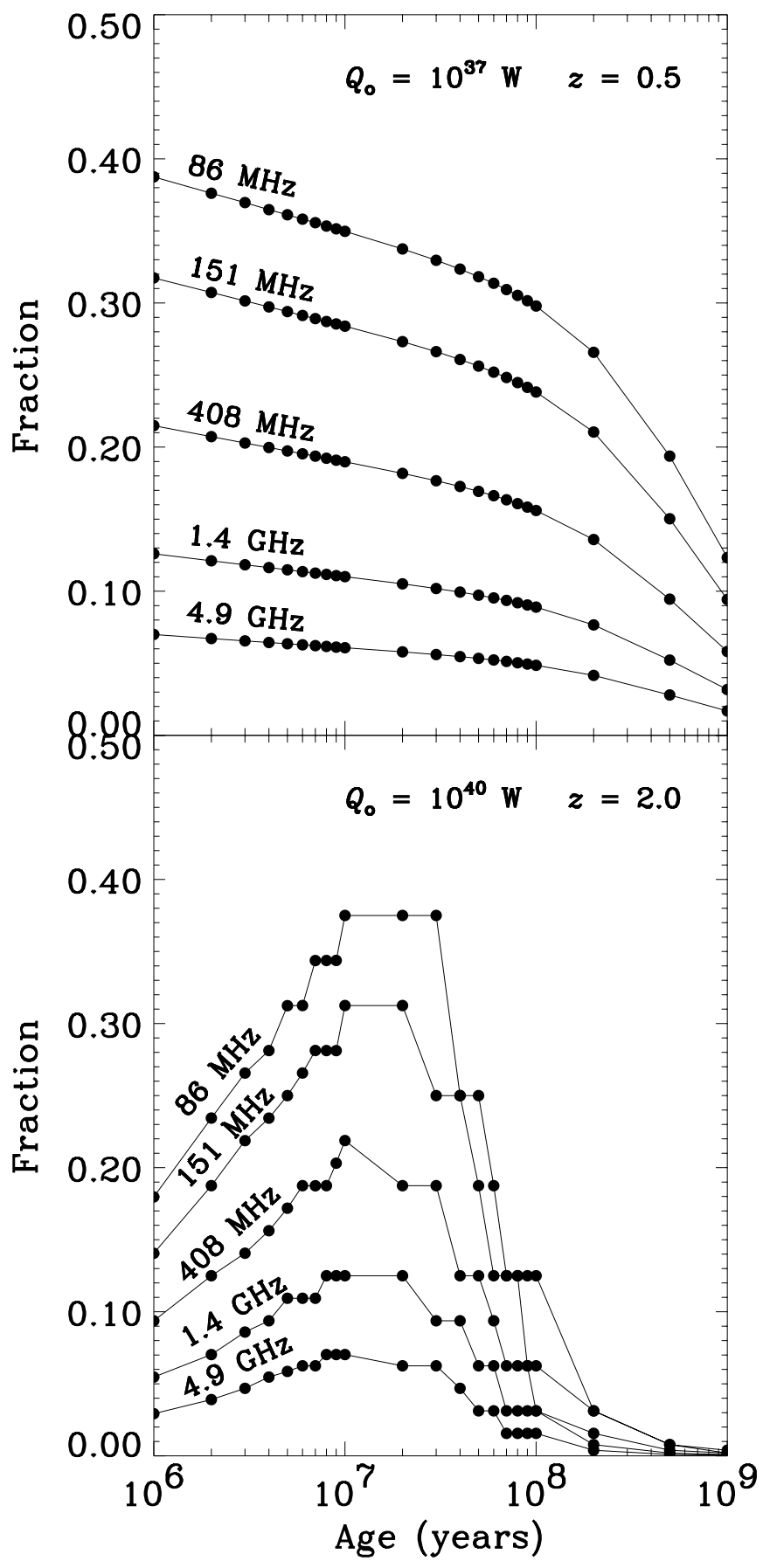

FIG. 1.-Plots showing the fraction of the age of the radio source for which the particles radiating at a certain frequency have been in the radio lobe vs. the age of the radio source. The bottom plot is for a simulated radio source with each jet carrying a bulk kinetic jet power $Q_{0}=10^{40} \mathrm{~W}$, and the top plot is for $Q_{0}=10^{37} \mathrm{~W}$. The assumed environment for these simulated radio sources has a number density at radius $100 \mathrm{kpc}$ of $n_{100}=$ $2 \times 10^{3} \mathrm{~m}^{-3}$ and a spherically symmetric profile outside a core radius of $r=10 \mathrm{kpc}$ whose density falls off as $r^{-1.5}$. The normalization factor $c_{1}$ for the source growth in the model of Falle (1991) is 3.5 (Kaiser et al. 1997).

particle now radiating in the lower $B$-field of $0.1 \mathrm{nT}$ at 5 $\mathrm{GHz}$ with $\gamma \sim 3 \times 10^{4}$ would, prior to the decrease in magnetic field strength, have had $\gamma \sim 10^{5}$.

Matthews \& Scheuer (1990) were the first to consider the losses suffered by particles of a given Lorentz factor $(\gamma)$ caused by synchrotron cooling and adiabatic expansion losses as a function of time and to quantify their influence on $d \gamma / d t$. Kaiser, Dennett-Thorpe, \& Alexander (1997) have modified their relation to include the influence of inverse Compton losses off the cosmic microwave background (which follow the behavior of synchrotron losses), and this may be used to relate the Lorentz factor of a synchrotron particle contributing to the emission at some given frequency, the time it was injected into the lobe, and its Lorentz factor at the time it was injected. The relation can be used to define a maximum time that can elapse between an initial time when a particle is injected with Lorentz factor $\gamma$ and the time of observation, if the particle is to emit synchrotron radiation at the specified observing frequency. This time of injection is called $t_{\min }$; prior to this time, even if particles of extremely high Lorentz factor are injected into the lobe, their enhanced energy losses will be so catastrophic that their Lorentz factors will be too low at the time of observation $^{2}\left(t_{\mathrm{obs}}\right)$ to contribute to radiation at the chosen frequency in a given $B$-field.

Thus for the ensemble of particles contributing to the radiation at a given emitted frequency at $t_{\mathrm{obs}}$, those that had the largest Lorentz factors at injection are injected at $t_{\text {min }}$ and those with the lowest are those actually injected at $t_{\mathrm{obs}}$. There is no trivial identity that connects $t_{\min }$ with the age of the source, as Figure 1 shows.

The clear implication from this figure is that a few times $10^{7} \mathrm{yr}$ is a very definite upper limit to the maximum radiative lifetime of a synchrotron-emitting particle, if the magnetic field experienced by the particles in the lobe is as we describe in $\S 9.2$ (namely, gently decreasing, because of the expansion of the lobe, and of order a few nT). Particles responsible for radiation at lower frequencies have a greater difference between $t_{\min }$ and $t_{\mathrm{obs}}$, as seen in Figure 1 and discussed in Blundell et al. (1999).

Thus, reconciling inferred spectral ages (discussed in $\S 2$ ) (particularly if synchrotron cooling is the only loss mechanism accounted for) with source ages (see $\S 5$ ) necessarily requires a rethink, since any given set of particles only contributes to the radiation for a small fraction of the age of a classical double. We discuss such a rethink in $\S 7$.

\section{SOURCE LIFETIMES}

Given current terminology in the literature we first clarify our usage of the terms "age" and "lifetime" in the context of observed radio sources. Knowledge of their lifetimes - the maximum duration of time for which sustained accretion and jet production is to be maintained - is relevant for constraining models of jet-producing central engines. Current modeling of the maximum duration of time for which sufficient jet power may be extracted from the black hole suggests limits of $10^{8}-10^{9} \mathrm{yr}$ (Meier 2000), which depend on both the mass and the spin of the black hole. Knowledge of the ages of the observed objects under study is crucial if one is to correctly interpret details of the environmental context in which the radio source resides.

\section{SOURCE AGES}

The simplest constraints on source ages come from making use of the measured projected physical size of a

\footnotetext{
${ }^{2}$ We use the term "time of observation" quite liberally here to mean "when the source is intercepted by our light-cone" or equivalently "when the light we ultimately observe leaves the source."
} 
radio galaxy in a particular cosmological model and estimating the speed at which it has expanded to that size.

Direct measurements of the advance speeds inferred from the proper motion of the hot spot in compact symmetric objects have been made by Owsianik, Conway, \& Polatidis (1998) and Owsianik \& Conway (1998), who find them to be rapid $(0.2 c-0.25 c)$. The model for source expansion of Falle (1991), where the sources expand into poor-group-type environments (e.g., as parameterized in Blundell et al. 1999; Blundell \& Rawlings 1999), predicts such speeds for the earliest stages of a source's life. This model predicts that these speeds reduce gradually by an order of magnitude as the sources age. We now consider speed estimates for these older classical double radio sources which are the focus of this paper. Many of these speed estimates have come from consideration of side-to-side asymmetries that are presumed to arise from light-travel time effects. These effects arise because the lobe nearer to the observer is seen at a more recent epoch than the other lobe, so it is seen when it is older and hence, in the absence of other influences, longer.

The faster a source is expanding the more likely it is to exhibit asymmetries in arm length, as expressed in equation (3) and as shown in the bottom plot of Figure 2. The observed asymmetry in arm length (quantified as $x$ ) of an intrinsically symmetric radio source expanding from the center of a spherically symmetric environment, each of whose lobe lengths is growing at speed $c \beta_{H}$ and whose jet axis is oriented at angle $\theta$ to the line of sight, is given by

$$
x=\frac{1-\beta_{H} \cos \theta}{1+\beta_{H} \cos \theta} .
$$

Longair \& Riley (1979) measured the arm-length asymmetries of classical doubles from the $3 \mathrm{C}$ catalog and, from the maximal asymmetries found, deduced that there was an upper limit of $0.2 c$ to the advance speeds of these objects. A similar and subsequent analysis by Banhatti (1980) found that half the classical double sources had advance speeds between 0.1c and 0.4c. More recently, Best et al. (1995) performed a similar analysis on objects from $3 \mathrm{C}$, but when they allowed for the misalignment of up to $10^{\circ}$ out of colinearity for the two arms of a radio source, they found that the distribution of arm-length asymmetries was best reproduced by a mean advance speed of $0.2 c$, with some advance speeds extending up to $0.4 c$. A more realistic analysis was performed by Scheuer (1995), who avoided attributing asymmetric arm lengths to light-travel time effects unless there was independent evidence of relativistic effects from the detection of a jet. Mindful that environmental asymmetries have been shown to cause significant asymmetries in arm lengths, ${ }^{3}$ we conclude that arm-length asymmetries that are caused by effects other than light-travel time effects must not be included in this type of analysis. Therefore, we adopt Scheuer's upper limit that the maximum advance speed of (fairly small and powerful) classical doubles is likely to be $0.03 c$. Such a speed, when taken together with the physical sizes of classical doubles (e.g., several hundreds of kiloparsecs) such as those studied by Alexander \& Leahy (1987), gives ages of over $10^{8} \mathrm{yr}$. These ages are an order of magnitude larger than the ages Alexander \& Leahy inferred

\footnotetext{
${ }^{3}$ McCarthy, van Breugel, \& Kapahi (1991) found that the side of a source with a shorter lobe was invariably found to be that with brighter emission-line gas.
}
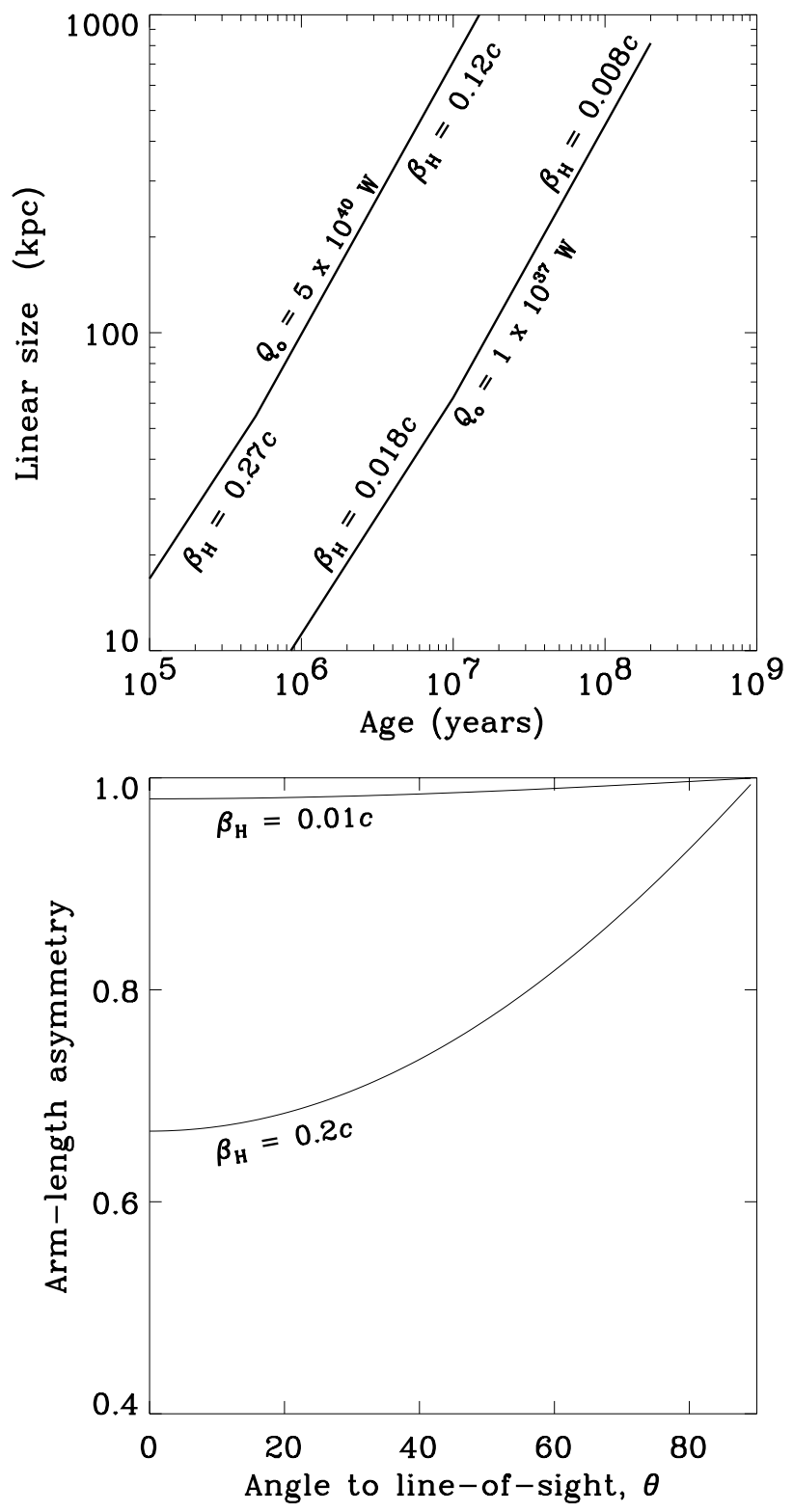

FIG. 2.-Top: Linear size of radio source (in kpc) vs. source age (in yr); the left curve is for a source each of whose jets carries a bulk kinetic power $Q_{0}=5 \times 10^{40} \mathrm{~W}$, and the right curve is for a source with $Q_{0}=1 \times 10^{37}$ W, derived according to the model of Falle (1991) and assuming the same environments as for Fig. 1. The higher powered source reaches a size of 1 Mpc after only $\sim 1.5 \times 10^{7} \mathrm{yr}$, while the lower powered source reaches this size after $\sim 3 \times 10^{8} \mathrm{yr}$. The faster expansion speed of the higher powered source should manifest itself in more dramatic side-to-side asymmetries due to light-travel time effects, as shown in the bottom plot. Bottom: Predicted arm-length asymmetries expected for two different example advance speeds.

by equating the spectral ages of the strips of lobe closest to the core with the age of the radio source.

The largest radio sources (the so-called giants, which have projected linear size $D>1 \mathrm{Mpc}$ ) show no indication of being larger because they have expanded more quickly ${ }^{4}$ than shorter radio sources: Ishwara-Chandra \& Saikia (1999) found for their sample of giant radio sources that

\footnotetext{
${ }^{4}$ Although examples of a distinct situation, rapidly expanding gigahertz-peaked spectrum sources associated with $\mathrm{Mpc}$ lobes are known; see, e.g., Schoenmakers et al. (1999).
} 
these are not significantly more asymmetric than sources with smaller physical sizes. If the giant radio sources do expand at speeds no faster than the value quoted above, then their large linear sizes must be achieved by higher ages, i.e., in excess of about a few times $10^{8} \mathrm{yr}$, potentially exacerbating the problem of source ages being highly in excess of the maximum radiative lifetime for synchrotron-radiating particles.

The spectral ages of giants studied by Mack et al. (1998) and Lacy et al. (1993) (all with radio luminosities below $10^{26}$ $\mathrm{W} \mathrm{Hz}{ }^{-1} \mathrm{sr}^{-1}$ and likely to have jet powers $\sim 10^{37} \mathrm{~W}$ ) were found to be (3-4) $\times 10^{7} \mathrm{yr}$, which if identified with their source ages would imply speeds of $\gtrsim 0.1 c$. Given the maximum speed limit identified by Scheuer (1995) for the most powerful classical doubles, taken at face value this would appear to go against the intuitively compelling idea (substantiated by the dimensional analysis of Falle 1991; see Fig. 2) that less powerful sources grow less quickly than more powerful sources.

\section{HOW FAR AWAY FROM THE HOT SPOT CAN THE LOBE MATERIAL RADIATE?}

Jenkins \& Scheuer (1976) pointed out that if synchrotron cooling played a part in the spectral shape of extended lobes, lobes should be observed to extend farther at low frequency than at high frequency. This rarely seems to be the case (Blundell et al. 2000). Rather, the extent of the lobe seems to depend on the luminosity of the radio source: low- $P$, large- $D$ sources (such as those studied by Alexander $\&$ Leahy 1987) invariably seem to have their lobe emission extending all the way back to the core. Evidence that this strong structural dependence on luminosity was the case was presented by Jenkins \& McEllin (1977), who identified that the more luminous classical doubles had a higher fraction of flux density originating in the outermost regions (within $15 \mathrm{kpc}$, i.e., the head and hot-spot regions) than lower luminosity sources. This is very pronounced in highredshift radio galaxies, where the emission seen at $\mathrm{GHz}$ frequencies comes very largely from the hot spot and very little from the lobes (see example maps of such high-redshift radio galaxies from the $6 C^{*}$ sample in Blundell et al. 1998). In contrast, those high- $P$, small- $D$ sources (such as those studied by Liu et al. 1992) seem to show lobes extending only, say, halfway back from the head to the core. (Although sometimes in these powerful sources with docked tails, faint emission can be still be seen close to the core [Leahy, Muxlow, \& Stephens 1989]. However, while Leahy et al. found this emission to be very faint, it was not found to be steep. Rather it was characterized by emission with a similar spectral index to that measured somewhat closer to the hot spot.)

Thus it is in the low-luminosity sources that the confrontation of radiative lifetime and source age is most problematic: $\mathrm{GHz}$ lobe emission in these objects, including at points farthest from the hot spot, is seen from synchrotronemitting particles with radiative lifetimes $\lesssim 10 \%$ of the source age.

\section{HOW TO REINTERPRET/RECONCILE SPECTRAL AGES}

There are two main approaches to the reinterpretation of spectral ages to reconcile them with source ages: (1) lowering the magnetic field throughout the lobe below the equipartition values traditionally used, so that the ages inferred from the spectral aging method are higher and more consis- tent with their true ages ( $\S 7.1$ ), and (2) mixing plasma of different ages and particularly mixing the plasma farthest from the hot spot with recently accelerated particles (this approach takes a number of different forms, and we discuss six of them in $\S \S 7.2-7.7$ ).

\subsection{Subequipartition B-Field in the Lobe Heads}

Alexander \& Pooley (1996) outline a means by which the expansion speed implied by the spectral aging analysis of Cygnus A might be made more than an order of magnitude lower in order to be consistent with its dynamically inferred expansion speed based on the assumption of ram pressureconfined hot spots. If the magnetic field strength in the lobe were below the value that would be in energy equipartition with the particle pressure, then the spectral ages ${ }^{5}$ would be higher and less discrepant with the dynamical ages than if equipartition magnetic field strengths were used. The expansion speed $v_{h}$ inferred from spectral aging depends on the assumed magnetic field strength $B$ as $v_{h} \propto$ $B^{3 / 2}$, and thus to reduce $v_{h}$ by a factor of $\gtrsim 10$ requires $B$ to drop by a factor of $\gtrsim 5$.

Alexander \& Pooley (1996) cited strong observational evidence for equipartition in the hot spots (Harris, Carilli, \& Perley 1994). ${ }^{6}$ They suggested that it is the process by which the plasma leaves the hot spot that causes the magnetic field strength to go out of equipartition with the energy in the particles. They aimed to model the flow out of the hot spot by steady diverging streamlines along which magnetic flux is conserved (i.e., the flux-freezing condition of $B \propto 1 / A$ holds, where $A$ is the cross-sectional area of some fixed loop) and along which mass is conserved (i.e., $\rho v A$ is constant, where $\rho$ and $v$ are the density and velocity along a streamline at some point). They obtain a relationship for the ratio of the energy densities in the particles $\left(u_{p}\right)$ and in the magnetic field $\left(u_{B}\right)$ in terms of the velocities along the streamlines. This ratio is derived using relationships for the pressure $p$ and density $\rho$ in terms of these velocities from Landau \& Lifshitz (1987, eq. [83.16]). They obtain the following:

$$
\frac{u_{p}}{u_{B}} \propto \frac{p}{B^{2}} \propto \frac{p}{\rho^{2} v^{2}} \propto \frac{1}{v^{2} / c_{*}^{2}} \frac{\left(1-v^{2} / 7 c_{*}^{2}\right)^{4}}{\left(1-v^{2} / 7 c_{*}^{2}\right)^{6}},
$$

where $c_{*}$ is the velocity at the point in the flow where $\rho v$ is a maximum. They approximate the velocity out of the hot spot to be $c_{*}$. Alexander \& Pooley claim that a drop in $v$ by a factor of $1 / \sqrt{2}$ readily gives the required increase in $p / B^{2}$ of a factor of 5. In fact their formula (shown here in eq. [4]) shows that to achieve this increase in $p / B^{2}$ requires the bulk flow velocity to decrease from its initial value by a factor of 0.4 .

\subsection{Fast Bulk Backflow}

In two spectral aging studies (Alexander \& Leahy 1987; Liu et al. 1992) the authors found that the spectral ages they derived implied advance speeds that were unacceptably high, even not accounting for adiabatic losses, in the light of

\footnotetext{
${ }^{5}$ Though note that this still ignores the consequences of expansion losses.

${ }^{6}$ Harris et al. detected X-ray emission associated with the hot spot that, if caused by the synchrotron self-Compton process, requires a magnetic field strength very close to the equipartition value given certain assumptions (specifically that there is no dominant presence of protons).
} 
the then upper limit ${ }^{7}$ to head advance speeds of Longair \& Riley (1979) of about $0.2 c$. As pointed out by Winter et al. (1980), the speed at which a given strip of plasma in the lobe separates from the hot spot is the antivector sum of the oppositely directed head advance speed and the backflow speed in the frame of the host galaxy. Bulk backflow speeds of about $0.1 c$, i.e., comparable to the advance speeds, were invoked to alleviate the requirement for such high advance speeds.

Leahy et al. (1989) find that the central regions of lobes in the higher powered sources have much less distortion than do those of lower powered sources. They suggest that this means that there is less backflow in more powerful sources. If the spectral age estimates were true this would be in contradiction with Alexander \& Leahy (1987) and Liu et al. (1992), who found that the lobe speed $v_{l}$ (sum of advance and backflow speeds deduced from spectral aging) increases with radio luminosity $P$ as $v_{l} \propto P^{0.33}$, assuming that the advance speeds are as deduced by Scheuer (1995; see $\S 5$ ).

A high bulk backflow speed requires some physical sink for the backflowing material, and a few examples of $\mathrm{X}$-shaped sources are known where this may be the case (Leahy et al. 1997; Dennett-Thorpe et al. 1999). However, there appears to be no evidence of powerful classical double radio sources showing any excess lobe structure indicative of backflow at low frequencies like $74 \mathrm{MHz}$ than at $\mathrm{GHz}$ frequencies (Kassim et al. 1993; Blundell et al. 2000). There are no examples known of lobe emission near the core betraying signs of compression (for example, by enhanced surface brightness compared with the rest of the lobe).

In the context of a leaking hot spot model and the complex magnetic field structure in the vicinity of the hot spot, it seems unlikely that as plasma emerges from the hot spot in the frame of the host galaxy it has a huge bulk flow away from the hot spot toward the core.

Although some simulations have shown rapid bulk backflow speeds (see, e.g., Norman et al. 1982), these speeds are strongly influenced by the boundary conditions used: open boundary conditions in the plane from which the jet emanates and through which lobe material may exit will enforce rapid flow away from the hot spot. Simulations with more appropriate boundary conditions can still show fast, but filamentary, backflow in the very early stages of a source's life. State-of-the-art simulations have much to offer in this area.

\subsection{Turbulent (Convective) Backflow}

A variant on the bulk backflow model (Leahy et al. 1989) is to assume that the turbulent mixing within the lobe of freshly injected plasma and older plasma is sufficiently large-scale and fast that efficacious transport of highly energetic particles to great distances away from the hot spot is achieved. In practice, this requires the effective velocity of transport to still be of the same order as the invoked bulk backflow speeds discussed in $\S 7.2$.

\subsection{Particle Reacceleration}

An alternative way of introducing highly energetic particles into regions of the lobe close to the core of a radio source is to invoke the possibility that the hot spot is not the only source of high- $\gamma$ particles, but that within the lobe itself reacceleration of particles occurs.

\footnotetext{
${ }^{7}$ See discussion of more up-to-date and tighter constraints in $\S 5$.
}

This has been invoked by Parma et al. (1999) in the context of FR I (Fanaroff \& Riley 1974) radio sources as a way to explain the low values of spectral ages in comparison with the dynamical ages. It has also been invoked by Carilli et al. (1991) in the case of Cygnus A to explain spectral shapes observed near the hot spot that do not exhibit symptoms of straightforward synchrotron losses.

Eilek \& Shore (1989) included in situ electron reacceleration both by Alfvén waves and by a Fermi mechanism in their model radio sources, which were evolving in a uniform medium. They found this gave little global influence on the luminosity behavior. This may be because in practice it is hard to achieve reacceleration mechanisms in the lobe, whose efficacy is comparable to that of the hot spot and the rate at which it is able to supply energized particles. It is even harder if one considers that the particle acceleration mechanism has to generate higher $\gamma$ particles than are needed to radiate in the higher magnetic field region of the lobe head, a point to which we return in $\S 10$. Independent evidence that particle reacceleration is not occurring has been found by Rudnick et al. (1994) and Katz-Stone \& Rudnick (1994) and is discussed in $§ 12$.

\subsection{Freezers}

Eilek, Melrose, \& Walker (1997) have considered possible physical mechanisms that would cause radio sources to appear younger than their implied dynamical ages. They invoked the idea that if a relativistic electron spends most of its time in a low-field region, and then diffuses into a highfield region in which it must radiate more efficiently than in a low-field region, on average it loses energy more slowly than if it were continuously in the high magnetic field region. ${ }^{8}$ If there are substantial inhomogeneities in the $B$-field and a sufficient quantity of energetic particles were somehow contained within a low magnetic field state, then this could in principle occur.

The filamentary nature of radio lobes seen in the best quality total intensity images (e.g., Cygnus A by Carilli et al. 1991) is circumstantial evidence that there are discrete regions of high and low magnetic fields within lobes. However, we note that it is only with the certainty of containment of the fast synchrotron particles that this could be a viable mechanism (see $\S 7.7)$.

\subsection{Low Pitch Angle Reservoir}

Spangler (1979) pointed out that electrons with small pitch angles do not suffer significant synchrotron losses but would diffuse in pitch angle owing to the influence of irregularities in the magnetic field he envisaged being superposed on a uniform field longitudinal with the lobe. He suggested that the loss via synchrotron cooling of energetic electrons in high pitch angle states is balanced by resupply from the low pitch angle reservoir. However, it is unlikely that the relative fraction of low pitch angle particles is sufficient to replenish the rather larger fraction of rapidly radiating high pitch angle electrons, as the fraction of particles having pitch angle less than $\theta$, for an isotropic distribution, is given by $1-\cos \theta$. Moreover, such a model relies on having an essentially longitudinal magnetic field, for which there is no observational evidence and which would be difficult to

\footnotetext{
${ }^{8}$ Such an idea of a "magnetic freezer" was first invoked by Scheuer (1989) in the context of low magnetic fields in jets to preserve an extremely high Lorentz factor population of particles from rapid synchrotron losses.
} 
sustain on theoretical grounds, because anchoring such magnetic flux would be very difficult.

\subsection{Fast Streaming}

We now consider whether relativistic particles can stream through low-energy plasma to penetrate those parts of the lobe whose original supply of energetic particles have long since radiated their energy away.

Streaming of high- $\gamma$ particles parallel to field lines is, of course, extremely fast and can proceed at speeds close to $c$. In contrast, motion perpendicular to the local field lines will be extremely slow. Since there is no observational basis for magnetic fields purely longitudinal with the lobe (see $\S 7.6$ ) and tangled fields (albeit on an unknown variety of scale lengths) are more likely, it is necessary to consider mechanisms for the scattering of particles to randomize their directions so that streaming parallel to the local field configuration may occur.

The anomalous diffusion mechanism of Rechester \& Rosenbluth (1978) involves the random walk of the particles taking place across randomly oriented regions of magnetic field. Following Duffy et al. (1995) and assuming a tangling scale of $10 \mathrm{kpc}$, in $10^{6} \mathrm{yr}$ the rms distance diffused is about a few hundred kiloparsecs. This distance is comparable to the arm lengths of the large- $D$, low- $P$ sources in the spectral aging study of Alexander \& Leahy (1987), and the timescale is less than the radiative lifetime of synchrotron-emitting particles in these sources $(\S 3)$.

Such scattering mechanisms are necessary to explain the difficulties of plasma containment seen in laboratory plasmas (Dendy 1993), and there is no basis for believing that astrophysical plasmas should not have the tangled configurations that cause the particles to be scattered.

Winter et al. (1980) claimed that there is an upper limit to the mixing speed of 4 times the advance speed, based on the assertion that, otherwise, a uniform population of electrons would be seen in all parts of the lobe; such an analysis neglects the influence of the magnetic field configuration (specifically the gradient in strength from hot spot to core) within the lobe, discussed in $\S 10$. The speeds that we discuss here should be regarded as penetration speeds rather than mixing speeds.

\section{ENERGY TRANSPORT AND THE RARITY OF DEAD RADIO GALAXIES}

It is clear that there are some serious drawbacks to the assumptions of a significantly subequipartition $B$-field ( 7.1 ; see also $\S 9.2$ ) within the lobe heads, fast bulk backflow ( $(7.2)$, convective backflow ( $§ 7.3$ ), and a low pitch angle reservoir $(\S 7.6)$.

However, the scenarios for replenishing the lobes with energetic particles long after the hot spot has passed by, outlined in $\S 7.4$ (particle reacceleration), $\S 7.5$ (freezers), and $\S 7.7$ (fast streaming), in principle can resolve any discrepancies implied by spectral aging. However, whatever the details of the physical mechanisms by which the scenarios discussed in $\S 7.4$ (particle reacceleration) and $\S 7.5$ (freezers) would be governed, it is not clear that either of these processes would know when to stop. In other words, there is potentially a problem with a surfeit of sources having highly radiant lobes long $\left(>10^{8} \mathrm{yr}\right)$ after jets and hot spots have switched off.

Examples of relic classical doubles are rare, with only a very few examples known (Cordey 1987; Harris et al. 1995;
Harris et al. 1993). Riley (1989) has used the sensitivity of the $6 \mathrm{C}$ survey to investigate whether any extended sources were unwittingly missed by the revised 3CR survey of Laing, Riley, \& Longair (1983). Apart from a few slight corrections due to measurement errors and confusion, no such sources were found. When Riley lowered the flux limit from $12 \mathrm{Jy}$ at $151 \mathrm{MHz}$ to $9.5 \mathrm{Jy}$, while the sample size increased by $50 \%$, all of these sources were found to be bona fide classical doubles. Giovannini et al. (1988) find that there are just $3 \%-4 \%$ of their sample of radio sources selected from the $\mathrm{B} 2$ and $3 \mathrm{C}$ catalogs that are relics.

In the example relic classical double studied by Cordey (1987), IC 2476, lobes of smooth extended low surface brightness emission are seen in images with greater than 0.5 resolution, which straddle a $z=0.027$ galaxy. Higher resolution images resolve out these lobes completely yet show no evidence of hot spots or a core or jet. It is very likely that at nonzero redshifts the absence of relic radio galaxies has its explanation in the youth-redshift degeneracy we described in Blundell \& Rawlings (1999). This is the effect in which a combination of declining luminosity with age for radio galaxies ${ }^{9}$ and the application of a survey flux limit means high-redshift radio galaxies are not seen at late stages in their lives. However, this explanation does not help to explain the deficit of relic radio galaxies in the local universe $(z<0.5)$, where the effect of the flux limit is not so drastic.

The expansion of the low- $\gamma$ plasma lobe can only occur on timescales consistent with the sound speed, so this cannot be a rapid process. A rapid decay time, however, does come from considering the brevity of the radiative lifetimes of the synchrotron-emitting particles.

We suggest that the role of fast streaming via anomalous diffusion of energetic particles throughout radio lobes might make a new contribution to the explanation for the rarity of "dead" radio sources found to date in surveys of the local universe. If there were a sudden cessation in the supply of bulk kinetic jet power, a radio source might begin to fade from view within a timescale comparable to the maximum radiative lifetimes of the synchrotron particles ( $\gtrsim 10^{6} \mathrm{yr}$ ) of the hot spot ceasing to inject high- $\gamma$ particles into the lobe. Though the magnetic field can still be present as long as the low- $\gamma$ plasma lobe is confined, high- $\gamma$ particles are no longer injected into the lobe to replenish the synchrotron-radiating population.

\section{A REFINED SPATIALLY RESOLVED MODEL OF RADIO SOURCES}

Up to now it has always been assumed that the high- $\gamma$ particles are the ones that govern the magnetic field and into which the magnetic field is frozen and that the localization of the high- $\gamma$ particles is cospatial with the magnetic field. This has yet to be justified in the context of astrophysical plasmas.

\subsection{Assumptions about Hot-Spot Output and the Nature of the Lobe}

We assume that the bulk backflow velocity out of the hot spot is very close to zero in the frame of the host galaxy. We

\footnotetext{
9 These arise in all reasonable models of radio galaxy evolution and environment, even while the jet continues to deliver a constant bulk kinetic power.
} 
picture the hot spot dumping successive elements of plasma. These elements relax into the lower pressure of the head region and, bringing a new supply of particles, the volume of the head region increases and the source expands.

In the radio source model we developed in Blundell et al. (1999), each element of hot spot material that transfers to the lobe heads has an energy distribution that is characterized by two adjoining power laws, the low-energy regime having a frequency spectral index ${ }^{10}$ of $\alpha=0.5$ and above the break frequency $v_{b}$ the frequency spectral index being $\alpha=1$. Where the break frequency occurs depends on the dwell time for which particles have resided in the enhanced magnetic field that has built up in the hot spot. Thus, with elements of the hot spot escaping into the lobe that have had different dwell times in the hot spot, a summation of spectra are injected into the lobe, which together comprise a curved injection spectrum. Note that such a model resolves the "injection index discrepancy" discussed by Carilli et al. (1991, § 5.2.2.2). Carilli et al. said that the low-frequency spectral index measured in the lobe should reflect either the $\alpha=0.5$ spectral index of the hot spot (if expansion losses were small) or the $\alpha \sim 1$ spectral index of the hot spot seen at higher frequencies (if the expansion losses were large). In fact they measure a low-frequency spectral index of 0.7 . We remark that the low-frequency spectral index should thus not be identified with the injection spectrum; this might lead one to spuriously conclude that the injection spectrum varies along the lobe and hence throughout the lifetime of a source.

These elements dumped into the lobe head, we suggest, may be identified with the turbulent eddies in the hot-spot model of De Young (2000) that have escaped out of the hot spot. As long as they are on sufficiently small spatial scales, these eddies will have magnetic field strengths in equipartition with their particle energies (De Young 2000). Equipartition of the magnetic energy density and the particle energy density is maintained in the hot-spot-to-head transition as long as the mixing - energized by the eddies from the hot spot-persists.

We assume that once an element of plasma is "dumped" by the hot spot it will gradually expand to the ambient pressure, that of the head. We regard this input of particles as constituting a new slab of the lobe whose addition lengthens the lobe. Subsequent injections of plasma into the lobe add further slabs, each of whose pressure is similar to that of the slabs to either side. Subsequent expansion of these volumes is entirely transverse since they are largely pressure matched front and back. If the preexpansion magnetic field is purely longitudinal, then its evolution is governed by $B \propto 1 / A$, whereas if it is tangled then it is approximated by $B \propto 1 / \sqrt{A}$. This causes a decline in the magnetic field strength, a point to which we return in $\S 9.2$.

The collection of many slabs constitutes a body of plasma that purveys the magnetic field. We posit that the slabs of plasma are dumped by the hot spot, and, once the radiative lifetime of their particles has expired, they will make no further direct contribution to the synchrotron radiation. However, their contribution is as a matrix of low- $\gamma$ material supplying a magnetic field that subsequently injected high- $\gamma$

\footnotetext{
${ }^{10}$ We use the convention for spectral index $(\alpha)$ that $S_{v} \propto v^{-\alpha}$, where $S_{v}$ is the flux density at frequency $v$.
}

particles penetrate (perhaps via the Rechester \& Rosenbluth 1978 mechanism; see § 7.7) and radiate in.

\subsection{How the Lobe Magnetic Field Evolves}

We have used our non-self-similar model for radio galaxy evolution (developed in Blundell et al. 1999) and refined it as in $\S 9.1$. We emphasize that in this model equipartition is preserved in the transfer of plasma from the hot spot to the head. At successive points throughout a source's lifetime we consider the volume of plasma injected into the lobe from the hot spot in the preceding $10^{3} \mathrm{yr}$. The length of this slab of plasma is taken as the distance advanced by the head of the source during that $10^{3} \mathrm{yr}$. We imagine that a slab of plasma, being surrounded on either side by slabs of plasma at very similar pressure, can expand only transversely into the intergalactic medium. Note that this is the same geometric configuration as envisaged by Chyży (1997). Transverse expansion is what governs the strength of the tangled magnetic field. With an assumed pressure gradient away from the head that falls off with the square of the distance (thus giving a ratio of pressure in the head to that halfway along the lobe of $\sim 4$, in agreement with Kaiser \& Alexander 1999), this simple model can give a drop in magnetic field of over an order of magnitude in megaparsecscale radio sources (see Fig. 3).

We note that the $B$-field strength in almost all of the most radiant regions of the lobe and head is nothing like the factor of 5 below equipartition required by Alexander \& Pooley (1996) to explain the spectral age discrepancy in Cygnus A. The region of the lobe nearest the core has the lowest $B$-field at $t_{\text {obs }}$; however, when the source was aged

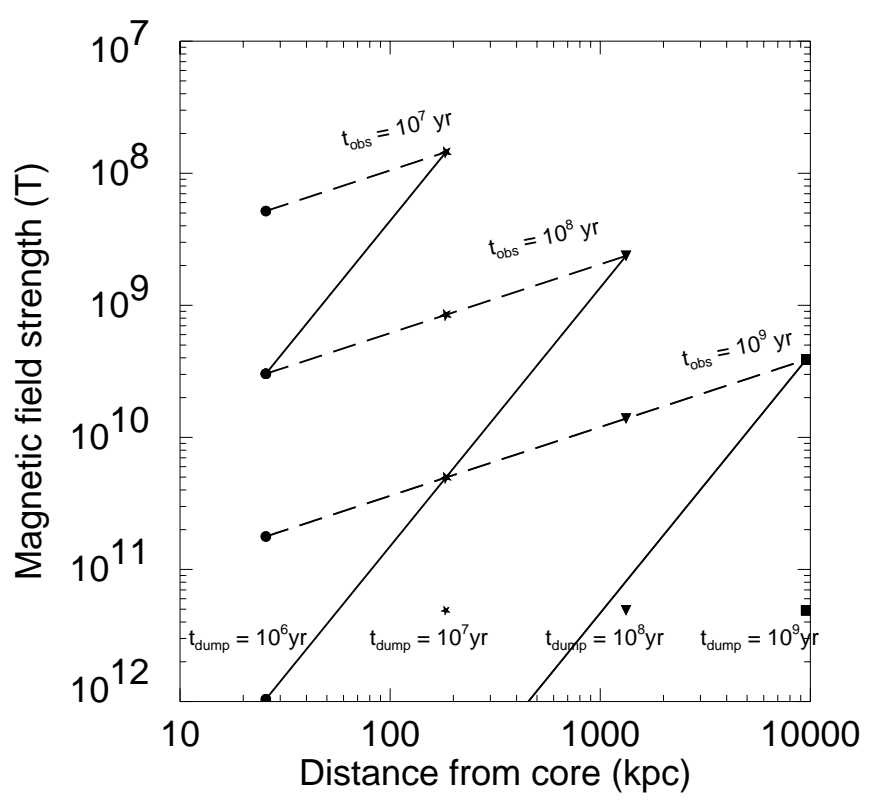

Fig. 3.- Magnetic field strength in a radio lobe at a given distance away from the core. The solid lines are calculated for the model described in $\S 9.2$, where flux freezing is invoked as the lobe expands transversely. The dashed lines show the magnetic field strength throughout the lobe if the field expands according to $B \propto 1 / \sqrt{A}$. The set of lines at the top shows the various magnetic field strengths if the source is observed aged $10^{7} \mathrm{yr}$, the middle set if the source is aged $10^{8} \mathrm{yr}$, and the bottom set if the source is aged $10^{9} \mathrm{yr}$. As the source ages the $B$-field falls. For each set of lines corresponds to a source with each jet carrying a bulk kinetic jet power $Q_{0}=5 \times 10^{39} \mathrm{~W}$. The same environmental parameters are used as for Fig. 1. 

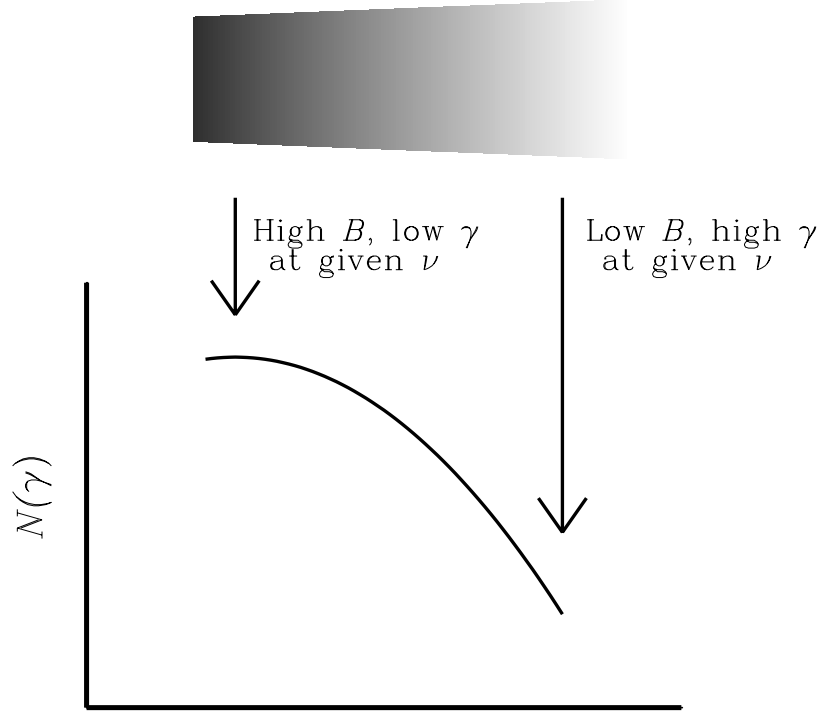

$\gamma$

FIG. 4.- Schematic illustration of the gently decreasing gradient in magnetic field strength along the lobe away from the head region and how for an observation at, say, $8 \mathrm{GHz}$, increasingly high Lorentz factor particles will be sampled in regions with lower magnetic field.

less than about a few times $10^{7} \mathrm{yr}$, the $B$-field of this plasma was roughly an order of magnitude higher (Fig. 3), and synchrotron cooling would have severely depleted the high- $\gamma$ particles injected simultaneously. Very low $B$-fields in the lobes near the core at $t_{\mathrm{obs}}$ cannot reconcile spectral and dynamical ages.

\section{SPECTRAL GRADIENTS}

Spectral gradients along the ridgelines of classical doubles, in the sense that spectra are steeper nearer the core than at the head of the lobe, are a common, though not ubiquitous, feature of these objects. An early example of a spectral gradient was found by Burch (1977) in 3C 452, where the spectral index between 1.4 and $5.0 \mathrm{GHz}$ was found to steepen from 0.7 near the head of the source to 1.7 near the center of the source. Burch attributed this behavior to increasing synchrotron losses with increasing distance from the core. The spectral steepening in the sources studied by Alexander \& Leahy (1987) at $\mathrm{GHz}$ frequencies was directly interpreted as increasing synchrotron losses with increasing time elapsed since injection from the hot spot. This assumption paved the way for their break frequency fitting and spectral aging analysis.

Cygnus A itself has been shown by Alexander, Brown, \& Scott (1984) to have lobe spectra that steepen away from the hot spot between frequencies of 1 and $5 \mathrm{GHz}$, with the spectral index reaching a maximum of about 2 in the most extended structure. They interpreted these variations as the results of synchrotron aging, making it possible to estimate the speed of advance of the hot spots. However, this same source has a spectral index gradient between 74 and 327 $\mathrm{MHz}$ (Kassim et al. 1996) of $\Delta \alpha \gtrsim 0.3$. It is hard to see that this low-frequency gradient could have the same origin as that which Alexander et al. (1984) suggested for the GHz regime.
Spectral gradients are seen to extend to sub-GHz frequencies in other examples as well (e.g., Jägers 1987, between $610 \mathrm{MHz}$ and $1.4 \mathrm{GHz}$ ).

If particles can diffuse distances comparable to lobe lengths in a few million years, then the constant replenishment of energetic particles suggests it is unlikely that synchrotron aging is the sole cause of the observed spectral steepening. Undoubtedly this plays a part in some short, very powerful source, however.

Wiita \& Gopal-Krishna (1990) have questioned both the assumption that the magnetic field is uniform throughout the lobe and the traditional usage of an estimate of the magnetic field strength in spectral aging analyses made in a region near the hot spot. ${ }^{11}$ They suggest that a lower $B$-field should be found closer to the core, rather than the higher "preexpansion" $B$-field found nearer the hot spot. Wiita \& Gopal-Krishna say that a lower magnetic field will result in a higher break frequency, and they use this fact to explain the "anomalous" spectral gradient in 3C 234 found by Alexander (1987). In this particular case, using their preferred $B$-field estimates gives spectral ages that are higher by a factor of only 1.5 than those of Alexander (1987).

We now consider the consequences for the spectral gradient of a magnetic field that declines along a lobe from the head toward the core, given the drop by about an order of magnitude shown in Figure 3. For a given observing frequency, say, $8 \mathrm{GHz}$, the emission arising from the higher magnetic fields near the head of the source will arise from lower Lorentz factor particles than the emission coming from lower magnetic fields closer to the core, which inevitably require higher Lorentz factor particles for radiation at the same frequency. If the underlying energy distribution of synchrotron particles is curved (an inevitable consequence of the leaky hot spot model described in $\S 9.1$ ), then the regions near the cores of these objects will show steeper spectra than those near the head by an amount $\Delta \alpha=$ $2 a_{2} \log \left(B_{\text {head }} / B_{\text {core }}\right)$, where $a_{2}$ is the coefficient of the curvature term in the frequency spectrum (cf. Blundell et al. 1999a) - see the schematic illustration in Figure 4.

Note that a spectral gradient will only be seen with a combination of a magnetic field gradient and a curved spectrum. There do exist sources with no spectral index gradient (see Jägers 1987). It may be that they have a magnetic field gradient, but the spectrum of particles supplied by the hot spot (e.g., because the hot spot has such a low $B$-field) is only described by $\alpha=1$, i.e., any break that may exist in the hot-spot spectrum is not seen.

Carilli et al. (1991) find that the bright filaments of Cygnus A have higher break frequencies than their environs, and throughout the source they find a correlation of total intensity and break frequency. We interpret this as confirmation that at a chosen observing frequency the higher $B$-field regions in a lobe will be "illuminating" a lower energy regime of the underlying particle distribution, which will have a flatter spectrum (hence higher "break") than the higher energy regimes illuminated by lower $B$ fields (see Fig. 4). This is strong evidence in favor of replenishment rather than static spectral aging, because it is the opposite effect to that predicted by synchrotron aging.

${ }^{11}$ The motivation for this previous usage is that this is a region in which the low-frequency spectral index should most reflect the injection index, but see the caveat about this in $\S 9$. 


\section{INDEPENDENT EVIDENCE FOR QUANTITATIVE MAGNETIC FIELD GRADIENTS}

We now consider quantitative estimates from the literature of magnetic field strengths and their comparisons with equipartition inferred values.

Murgia et al. (1999) have found for the very young (age $\lesssim$ $10^{5} \mathrm{yr}$ ) sources they studied that the values of equipartition magnetic field strength they derive are consistent with the magnetic field strengths responsible for the low-frequency turnovers via the synchrotron self-absorption mechanism.

Harris et al. (1994) show that the magnetic field required to give synchrotron emission observed in the hot spot (where the number of particles has been normalized by interpreting the X-ray emission from the hot spot as synchrotron self-Compton, i.e., it is the photons emitted by the synchrotron electrons that are upscattered) is very close to the equipartition value inferred in the usual way.

Farther removed from the hot spot, X-ray emission at approximately $2 \mathrm{keV}$ has been detected associated with radio lobes, which is likely to be cosmic microwave background (CMB) photons upscattered by synchrotron particles with Lorentz factors of about 1000, as follows:

1. Feigelson et al. (1995) and Kaneda et al. (1995) independently find for Fornax A that the ratio of the X-ray luminosity from the inverse Compton upscattered $\mathrm{CMB}$ photons taken together with the synchrotron emission from the lobes imply a magnetic field strength very close to that which is inferred from the usual equipartition arguments.

2. For 3C 326, Tsakiris et al. (1996) have inferred magnetic fields that are the same as, or lower by a factor of 2 than, the magnetic field strength derived from the usual equipartition calculation on the basis of $\mathrm{X}$-ray emission detected near the heads.

3. Subsequently for Cen B, Tashiro et al. (1998) found that the magnetic field strength implied by interpreting the $\mathrm{X}$-ray emission as upscattered CMB photons gives a magnetic energy 4 times lower than the particle energy. Such a wide discrepancy is most pronounced in the farthest regions from the hot spot, while at the hot spots themselves, equipartition is in fact preserved.

\subsection{Pressure and Magnetic Field Constraints near the Core}

We now turn our attention to independent calibration of the pressure in the lobes. Leahy \& Gizani (2000) have found that there is a considerable discrepancy with the value of the lobe pressure inferred from the usual equipartition argument and that obtained by fitting a King model to the profile of the cluster X-ray emission surrounding Her A, assuming a temperature and deducing the pressure that is confining the lobes. This is in the sense that the lobe pressure appears to be an order of magnitude too low for pressure balance with the external confining medium. Leahy \& Gizani (2000) conclude that some nonradiating, low-energy contribution (such as protons or very low $\gamma$ particles) must be responsible for the extra pressure. There is considerable independent evidence that very low $\gamma$ particles must be present in the lobes: (1) They are present in the jets that feed the lobes (as required by the details of the circular polarization detections of Wardle et al. 1998). (2) They are an inevitable consequence of the synchrotron decay process. Moreover, if it is the case that the particles that emit the observed synchrotron radiation represent a high- $\gamma$ tail that has penetrated the low- $\gamma$ matrix, then it is clear that the standard minimum energy calculation based on this emission, including that near the core, has a very unsound basis.

For a lobe with a particle reservoir whose energy distribution is given by

$$
N(\gamma) d \gamma=K \gamma^{-(2 \alpha+1)} d \gamma
$$

between all particle energies from $\gamma_{\min }$ to $\gamma_{\max }$ residing in mean magnetic field strength $\bar{B}$ over volume $V$ (assuming a filling factor of unity and no significant contribution from protons), the total energy budget is given by

$$
E_{\mathrm{tot}}=V K m_{e} c^{2} \ln \left(\frac{\gamma_{\max }}{\gamma_{\min }}\right)+\frac{\bar{B}^{2} V}{2 \mu_{0}},
$$

if $\alpha=0.5$, and, otherwise,

$$
E_{\mathrm{tot}}=V K m_{e} c^{2}\left(\frac{1}{\gamma_{\min }^{2 \alpha-1}}-\frac{1}{\gamma_{\max }^{2 \alpha-1}}\right)+\frac{\bar{B}^{2} V}{2 \mu_{0}} .
$$

For the case where $\alpha=0.5$, the energy budget is governed by the upper limit to the energy distribution, but given that no known lobes have spectral indices as flat as this, we do not consider this case further. For steeper spectral indices equation (7) shows us that this depends essentially not at all on $\gamma_{\max }$ (hereafter we neglect this term) but is governed by $\gamma_{\min }$. A choice of $\gamma_{\min }=50$ will underestimate this contribution to the energy budget by a factor of, e.g., $50^{0.2}$ for $\alpha=0.6$ if the true value is $\gamma_{\min }=1$.

On the naive assumption that at least for low energies the distribution can be represented by a power law, the other major unknown in this is the normalization of the number density of particles, $K$. This has been estimated in a couple of cases by inferring the number densities of the low- $\gamma$ particles required to inverse Compton scatter photons from the active galactic nucleus (AGN) radiation field up to the soft X-rays ${ }^{12}$ in the cases of 3C 219 (Brunetti et al. 1999) and Cen B (Tashiro et al. 1998). In the former case, Brunetti et al. (1999) found that the number density of particles must exceed the equipartition value by a factor of 10 (this is derived assuming $\gamma_{\min }=50$ ). In the latter case, Tashiro et al. (1998) assume that $\gamma_{\min }=1000$, which, given the evidence of inverse Compton scattering off the AGN radiation field, denies the presence of the $\gamma \sim 100$ particles responsible and is probably not the best choice of $\gamma_{\text {min }}$ given the evidence discussed earlier in this section. Brunetti et al. (1999) recalculate the energy budget for Tashiro et al.'s data using $\gamma_{\min }=50$ and find that a number density about 50 times higher than implied by the equipartition value is required to explain their X-ray detections.

The observed synchrotron luminosity $P_{v}$ is given by

$$
P_{v} \propto V K \overline{B^{1+\alpha}} v^{-\alpha} .
$$

The unknowns in this equation are famously $K$ (the normalization of the number density) and $\bar{B}$ (the mean magnetic

\footnotetext{
${ }^{12}$ We note in passing that it is possible to misconstrue extended X-ray emission from powerful high- $z$ classical doubles, which has been upscattered from the powerful quasar radiation field as arising from surrounding cluster emission. Accurate morphological information from highresolution observations with Chandra will be a great asset.
} 
field strength), although the product $K \overline{B^{1+\alpha}}$ is, of course, determined. If $K$ is found to be about 10 times higher than equipartition values, then the magnetic field strength giving rise to the observed luminosity is lower by a factor of approximately $10^{1 /(1+\alpha)}$ than the traditional equipartition calculation implies. Note that the constraints in number density from Brunetti et al. (1999) and Tashiro et al. (1998) pertain to emission that is close to the core, i.e., in the oldest regions of the lobes, precisely those regions predicted by our simple model of $\S 9.2$ to be those for which the synchrotronemitting population is most poorly approximated as a smooth continuation from the low- $\gamma$ population.

These results on the independent determination of magnetic field strengths (for the hot spots, heads, and lobes near the core) are thus in accordance with our simple model. The fast-streaming picture of $\S 7.7$ means that the spectral shape of the high- $\gamma$ population can be preserved throughout the lobe, but the normalization (i.e., $K$ ) will depend on the details of the diffusion mechanism. At the heads of the lobes, the plasma is being viewed within a timescale shorter than the synchrotron radiative lifetime, so the high- $\gamma$ population is a smooth continuation of the low- $\gamma$ population. However, near the core the normalization of the high- $\gamma$ population could substantially underestimate the normalization of the low- $\gamma$ population.

There are some very important implications of our inference in this section that the lobe pressures are roughly an order of magnitude larger than those estimated by the standard minimum energy arguments. We have already considered this possibility in Willott et al. (1999). In the notation of that paper, $f$ is the factor (greater than unity) that when multiplied by the minimum energy value (calculated in the traditional way) gives the total power delivered by the jets since they first switched on (see eq. [4] in that paper) and has a value of about 20 . This value is made up of several multiplying factors such as $f_{\min }$ (which accounts for an excess energy in the particles compared with that in the magnetic field), $f_{\text {geom }}$ (which accounts for the unknown deprojection of a radio source onto the plane of the sky), $g_{\text {exp }}$ (which allows for work done by the expansion of the radio source against the external medium), and $g_{\mathrm{ke}}$ (which accounts for energy in the bulk backflow of the lobe). Our inference in this section is that the bulk of the $f$-factor is contributed by $f_{\min } \sim 10$, leaving room for only much smaller contributions from $g_{\text {exp }}$ (probably $\sim 2$ ), $f_{\text {geom }}$, etc. There is therefore no room for major contributions to the pressure from protons or for the filling factor to be much lower than 1. Furthermore, the greater total energy in the lobes changes the normalization of the inferred jet power. Willott et al.'s (1999) Figure 7 shows that the effect of this change is to suggest that the bulk power in the jets is then of the same order as the power radiated away by the accreting central engine. This rough equality of kinetic and radiated outputs of an accreting black hole is a prediction of some models (Falcke, Malkan, \& Biermann 1995).

\section{DISCUSSION OF KATZ-STONE \& RUDNICK RESULTS}

Rudnick et al. (1994) and Katz-Stone \& Rudnick (1994) have made a new type of analysis of the high-fidelity imaging data of Carilli et al. (1991) on Cygnus A. They found evidence for a universal (curved) spectrum that describes emission over the entire source. That is, they take the spectrum at various points throughout the source, and then, on the $\log$ (flux density) versus $\log$ (frequency) plane, they perform translations to the different spectra that are parallel to either one or other axis. They found that these translations meant that all these spectra would overlie one another; i.e., they had precisely the same shape. This result has come from high-resolution images, whereas lower resolution images can give the impression of power-law spectra. We note that the most recent detections of the hot spot in Cygnus A in the submillimeter, by Robson et al. (1998) with SCUBA, imply a power-law spectrum with spectral index of roughly 1 , but with the comparatively low angular resolution of SCUBA $\left(\sim 15^{\prime \prime}\right.$ at $\left.850 \mu \mathrm{m}\right)$, it is likely that these spectra will be flattened by the contribution of the other hot-spot components in the SCUBA beam.

Katz-Stone \& Rudnick found no evidence for the evolution of the electron energy distribution function. Neither did they find a distribution resembling the Kardashev (1962) and Pacholcyzk (1970) model or the Jaffe \& Perola (1973) model; nor do they find any evidence of the traditionally assumed injection power-law distribution.

The translations of the spectra performed by Rudnick and Katz-Stone et al. are effectively moving around in age$B$-field space (see Katz-Stone \& Rudnick 1994). The curve they find is telling us, albeit indirectly, about the underlying particle distribution (which is what the hot spot injects). The universality of the spectrum found for Cygnus A strongly suggests that no particle acceleration processes have taken place at any significant level, since the shape of the original spectrum would not be preserved by such a process. Under these circumstances it is not a surprise that the spectra should be the same in the lobes and the hot spots. This argues for a constant-injection scenario (as in the hot-spot model of Blundell et al. 1999) throughout the fraction of a source's life comparable to or greater than the radiative lifetimes of the synchrotron-emitting particles.

The inclusion of fast streaming of high- $\gamma$ particles via anomalous diffusion helps to explain why Rudnick et al. (1994) see the universal spectrum: all parts of the lobe show us the energy distribution of particles as they were when (or within a few times $10^{6}-10^{7} \mathrm{yr}$ of being) first injected from the hot spot.

A lobe cut into a sequence of strips will not just be telling us about a sequence of differences arising just from the time of injection (as is inherently assumed in the spectral aging analyses) but mainly about different and decreasing magnetic fields. If fast streaming does occur, then this would be convolved with different Lorentz factors from different times of injection.

\section{CONCLUDING REMARKS}

The spectral aging method can work well as long as the ages to be measured are much shorter than the radiative lifetimes of the particles that constitute the spectrum and when the history of the magnetic field is well approximated. This has recently been demonstrated by Owsianik et al. (1998), Owsianik \& Conway (1998), and Murgia et al. (1999) for sources with ages $10^{2}-10^{3}$ and $10^{5} \mathrm{yr}$, respectively. In the former cases, direct verification of the advance speeds via proper-motion measurements of the hot spot with the VLBA is available and shows impressive agreement with the spectral ages of a similar object derived by Readhead et al. (1996). It is interesting in this regard that rather more convincing broken spectra and spectral breaks are found in these cases than for the spectra extracted from the much older classical doubles. 
The possibility of replenishment of high- $\gamma$ particles via diffusive streaming means that spectral ages in older and larger classical doubles are even more meaningless than previous worries have suggested.

The best way of viewing radio lobe emission is via an evolving magnetic field mediated by a low- $\gamma$ matrix, which, according to its strength and the observing frequency, will illuminate some small part of the electron energy distribution as recently injected by the hot spot. Such an approach is supported by the observations of Carilli et al. (1991) that the bright filaments of Cygnus A have flatter spectra than the surrounding fainter emission.

K. M. B. thanks the Royal Commission for the Exhibition of 1851 for a Research Fellowship. We are very grateful to Richard Dendy, Peter Duffy, and David De Young for helpful discussions, and especially to Robert Laing and the referee, Larry Rudnick, for a careful reading of the manuscript.
Alexander, P. 1987, MNRAS, 225, 27

Alexander, P., Brown, M. T., \& Scott, P. F. 1984, MNRAS, 209, 851

Alexander, P., \& Leahy, J. P. 1987, MNRAS, 225, 1

Alexander, P., \& Pooley, G. 1996, in Cygnus A: Study of a Radio Galaxy, ed. C. L. Carilli \& D. E. Harris (Cambridge: Cambridge Univ. Press), 149

Banhatti, D. G. 1980, A\&A, 84, 112

Best, P. N., Bailer, D. M., Longair, M. S., \& Riley, J. M. 1995, MNRAS, 275, 1171

Blundell, K. M., \& Alexander, P. 1994, MNRAS, 267, 241

Blundell, K. M., \& Rawlings, S. 1999, Nature, 399, 330

Blundell, K. M., Rawlings, S., Eales, S. A., Taylor, G. B., \& Bradley, A. D. 1998, MNRAS, 295, 265

Blundell, K. M., Rawlings, S., \& Willott, C. J. 1999, AJ, 117, 677

Blundell, K. M., Rawlings, S., Willott, C. J., Kassim, N. E., \& Perley, R. A. 2000, NewA Rev., in press

Brunetti, G., Comastri, A., Setti, G., \& Comastri, A. 1999, A\&A, 342, 57

Burch, S. F. 1977, MNRAS, 180, 623

Carilli, C. L., Perley, R. A., Dreher, J. W., \& Leahy, J. P. 1991, ApJ, 383, 554

Chyży, K. T. 1997, MNRÄS, 289, 355

Cordey R. A. 1987, MNRAS, 227, 695

Dendy, R. 1993, Plasma Physics: An Introductory Course (Cambridge: Cambridge Univ. Press)

Dennett-Thorpe, J., Bridle, A. H., Laing, R. A., \& Scheuer, P. A. G. 1999, MNRAS, 304, 271

De Young, D. 2000, NewA Rev., in press

Duffy, P., Kirk, J. G., Gallant, Y. A., \& Dendy, R. O. 1995, A\&A, 302, L21

Eilek, J. A \& Shore, S. N. 1989, ApJ, 342, 187

Eilek, J. A., Melrose, D. B., \& Walker, M. A. 1997, ApJ, 483, 282

Falcke, H., Malkan, M. A., \& Biermann, P. L. 1995, A\&A, 298, 375

Falle, S. A. E. G. 1991, MNRAS, 250, 581

Fanaroff, B. L., \& Riley, J. M. 1974, MNRAS, 167, 31P

Feigelson, E. D., Laurent-Muehleisen, S. A., Kollgaard, R. I., \& Fomalont, E. D. 1995, ApJ, 449, L149

Giovannini, G., Feretti, L., Gregorini, L., \& Parma, P. 1988, A\&A, 199, 73

Harris, D. E., Carilli, C. L., \& Perley, R. A. 1994, Nature, 367, 713

Harris, D. E., Stern, C. P., Willis, A. G., \& Dewdney, P. E. 1993, AJ, 105, 769

Harris, D. E., Willis, A. G., Dewdney, P. E., \& Batty, J. 1995, MNRAS, 273, 785

Ishwara-Chandra, C. H., \& Saikia, D. J. 1999, MNRAS, 309, 100

Jägers, W. J. 1987, Ph.D. thesis, Univ. Leiden

Jaffe, W. J., \& Perola, G. C. 1973, A\&A, 26, 423

Jenkins, C. J., \& McEllin, M. 1977, MNRAS, 180, 219

Jenkins, C. J., \& Scheuer, P. A. G. 1976, MNRAS, 174, 327

Jones, T. W., Ryu, D., \& Engel, A. 1999, ApJ, 512, 105

Kaiser, C. R., \& Alexander, P. 1999, MNRAS, 305, 707

Kaiser, C. R., Dennett-Thorpe, J., \& Alexander, P. 1997, MNRAS, 292, 723

Kaneda, H., et al. 1995, ApJ, 453, L13

Kardashev, N. S. 1962, Soviet Astron., 6, 317

Kassim, N. E., Perley, R. A., Carilli, C. L., Harris, D. E., \& Erickson, W. C. 1996, in Cygnus A: Study of a Radio Galaxy, ed. C. L. Carilli \& D. E. Harris (Cambridge: Cambridge Univ. Press), 182

Kassim, N., Perley, R. A., Eriksen, W., \& Dwarakanath, K. S. 1993, AJ, 106,2218

\section{REFERENCES}

Katz-Stone, D. M., \& Rudnick, L. 1994, ApJ, 426, 116

Lacy, M., Rawlings, S., Saunders, R., \& Warner, P. J. 1993, MNRAS, 264, 721

Laing, R. A., Riley, J. M., \& Longair, M. S. 1983, MNRAS, 204, 151

Landau, L. D., \& Lifshitz, E. M. 1987, Fluid Mechanics (2d ed.; Oxford: Pergamon)

Leahy, J. P., Black, A. R. S., Dennett-Thorpe, J., Hardcastle, M. J., Komissarov, S., Perley, R. A., Riley, J. M., \& Scheuer, P. A. G. 1997, MNRAS, 291,20

Leahy, J. P., \& Gizani, N. A. B. 2000, NewA Rev., in press

Leahy, J. P., Muxlow, T. W. B., \& Stephens, P. W. 1989, MNRAS, 239, 401

Liu, R., Pooley, G., \& Riley, J. M. 1992, MNRAS, 257, 545

Longair, M. S., \& Riley, J. M. 1979, MNRAS, 188, 625

Mack, K.-H., Klein, U., O’Dea, C. P., Willis, A. G., \& Saripelli, L. 1998, A\&A, 329, 431

Matthews, A. P., \& Scheuer, P. A. G. 1990, MNRAS, 242, 616

McCarthy, P. J., van Beugel, W., \& Kapahi, V. K. 1991, ApJ, 371, 478

Meier, D. 2000, NewA Rev., in press

Murgia, M., Fanti, C., Fanti, R., Gregorini, L., Klein, U., Mack, K.-H., \& Vigotti, M. 1999, A\&A, 345, 769

Myers, S. T., \& Spangler, S. R. 1985, ApJ, 291, 52

Norman, M. L., Winkler, K.-H. A., Smarr, L., \& Smith, M. D. 1982, A\&A, 113,285

Owsianik, I., \& Conway, J. E. 1998, A\&A, 337, 69

Owsianik, I., Conway, J. E., \& Polatidis, A. G. 1998, A\&A, 336, L37

Pacholczyk, A. G. 1970, Radio Astrophysics (New York: Freeman)

Parma, P., Murgia, M., Morganti, R., Capetti, A., de Ruiter, H. R., \& Fanti, R. 1999, A\&A, 344, 7

Readhead, A. C. S., Taylor, G. B., Xu, W., Pearson, T. J., Wilkinson, P. N., \& Polatidis, A. 1996, ApJ, 460, 612

Rechester, A. B., \& Rosenbluth, M. N. 1978, Phys. Rev. Lett., 40, 38

Riley, J. M. 1989, MNRAS, 238, 1055

Robson, E. I., Leeuw, L. L., Stevens, J. A., \& Holland, W. A. 1998, MNRAS, 301, 935

Rudnick, L. 2000, NewA Rev., in press

Rudnick, L., Katz-Stone, D. M., \& Anderson, M. C. 1994, ApJS, 90, 955

Scheuer, P. A. G. 1989, in Hot Spots in Extragalactic Radio Sources, ed. K. Meisenheimer \& H.-J. Röser (New York: Springer), 159 1995, MNRAS, 277, 331

Scheuer, P. A. G., \& Williams, P. J. S. 1968, ARA\&A, 6, 321

Schoenmakers, A. P., de Bruyn, A. G., Röttgering, H. J. A., \& van der Laan, H. 1999, A\&A, 341, 44

Siah, M. J., \& Wiita, P. J. 1990, ApJ, 363, 411

Spangler, S. R. 1979, ApJ, 232, L7

Tashiro, M., et al. 1998, ApJ, 499, 713

Tribble, P. C. 1993, MNRAS, 261, 57

Tsakiris, D., Leahy, J. P., Strom, R. G., \& Barber, C. R. 1996, in IAU Symp. 175, Extragalactic Radio Sources, ed. R. D. Ekers, C. Fanti, \& L. Padrielli (Dordrecht: Kluwer), 256

Wardle, J. C. F., Homan, D. C., Ojha, R., \& Roberts, D. H. 1998, Nature, 395,457

Wiita, P. J., \& Gopal-Krishna. 1990, ApJ, 353, 476

Willott, C. J., Rawlings, S., Blundell, K. M., \& Lacy, M. 1999, MNRAS, 309,1017

Winter, A. J. B., et al. 1980, MNRAS, 192, 931 